\title{
Choice-Process Satisfaction: The Influence of Attribute Alignability and Option Limitation
}

\author{
Shi Zhang \\ University of California, Los Angeles \\ and \\ Gavan J . Fitzsimons \\ University of Pennsylvania
}

This research investigates how choice-process satisfaction is influenced by limitation of choice option and by the types of features used to represent the options. Studies of choice satisfaction have focused on how satisfied the decision maker feels about the choice that has been made and have overlooked the importance of the process through which the decision maker makes a choice, i.e., choice-process satisfaction. We show that the comparability of choice options through alignable features increases choice-process satisfaction, whereas option limitation (i.e., making one option unavailable from a set of equally attractive options) decreases choice-process satisfaction. Further, this decrease in satisfaction, relative to all options being available, occurs for people who are given a set of options in which the difference features are alignable (i.e., differences of a corresponding dimension) but not for people who are given a set of options in which the difference features are nonalignable (i.e., differences of unique dimensions). We propose that alignable differences are easier to compare and have more weight in people's attribute processing, and thus give rise to a perception of a greater amount of information about the option set that is relevant for choice.

Theauthors gratefully acknowledge financial support by the Marketing Studies Center at UCLA; comments on an earlier version from Aimee Drolet, Ellie Fang, Art Markman, Atanu Sinha, the hel pful editor, and reviewers; and technical assistance from Carolyn Cohen, J eff Robinson, Laura Brown, and Noelle Triaureau. They also thank Bill Yost for recruiting participants from his class. The order of authorship is random.

Address correspondence and reprint requests to Shi Zhang, 110 Westwood Plaza B412, UCLA-Anderson School, Los Angeles, CA 90095. Fax: (310) 206-7422. E-mail: shi.zhang@ anderson.ucla.edu. 


\begin{abstract}
Making an option unavailable in this case would have a bigger impact than in a situation in which all options have nonalignable differences. Nonalignable differences are difficult to process and are less likely to make people aware that there is very much information about the options for decision making. This explanation and the interaction effect between option limitation and feature alignability are tested in four experiments. $\odot 1999$ Academic Press
\end{abstract}

\title{
INTRODUCTION
}

The issue of decision satisfaction is an important topic that has received scant attention in psychology and decision research. The few works that exist on this topic have focused either on the analysis of satisfaction in terms of postchoice dissonance reduction (F estinger, 1957) or more recently on the postchoice evaluations of a decision that produces conflicting feelings of satisfaction or regret (Houston, Sherman, \& Baker, 1991). Decision satisfaction, in these works, refers to the level of feelings of satisfaction or regret regarding the chosen alternative or rejected alternative. Satisfaction, therefore, is associated only with the decision about the outcome choice. The current research expands the study of decision satisfaction by focusing on the decision process itself rather than the choice outcome. The research attempts to isolate factors that may influence decision-process satisfaction and proposes a mechanism that accounts for the main and interactive effects of these factors.

The process through which a decision or choice is made constitutes an important aspect of decision satisfaction. People's affective experience of the decision process can be influenced by variables that shape the situation in which the decision occurs. For example, people can find themselves in a situation in which one of the choice options they are comparing is not available (e.g., the display shoe is out of stock in a shoe store). If a choice is required, they must therefore make a selection from the remaining options. Compared with a situation in which all the choice options are available, the former situation should make people feel that the "option to choose" is restricted, even when the unavailable option would not be their ultimate choice. Thus, they may be less satisfied with the experience of making a choice (e.g., Brehm, 1966). Researchers in legal and organizational justice domains have devoted considerable energy to the subject of procedural justice-perceived fairness of the justice process itself, independent of the outcome of the deliberation (Sheppard, Lewicki, \& Minton, 1992; Thibault \& Walker, 1975). We draw an analogy to the choice domain and examine decision makers' satisfaction with the decision process rather than the choice outcome, henceforth choi ce-process satisfaction.

The degree of satisfaction with the choice process is an important topic for investigation for both theoretical and practical purposes. Choice-process satisfaction may affect willingness to shop at a store at which the choice was made. For example, unsatisfactory experience with the choice process, such as unavailability of certain choice options, can generate significant customer 
complaints (e.g., Andersen Consulting, 1996; Hess \& Gersner, 1987). Furthermore, customers are found to be generally more satisfied, which implies a higher likelihood of repurchase, when they are given quality and reliability information about the choice options during their decision processes (Westbrook, Newman, \& Taylor, 1978). M oreimportantly, understanding what factors contribute to choice-process satisfaction may further illuminate the relation between comparison processes and postdecisional affective response in general. For example, Houston et al. (1991) provide evidence supporting a negative relationship between prechoice conflict due to the features of the alternatives and postchoice satisfaction. They demonstrate that alternatives with unique bad features generate conflicts and vacillation between alternatives. Once a choice is made, choice satisfaction is greater when the comparison and processing focus is on the rejected alternative (vs the accepted alternative). This occurs because if the rejected al ternative is focused on, its unique bad features will become especially apparent, and the unique bad features of the accepted alternative will be neglected (see Tversky, 1977). In contrast, if the processing focus is on the accepted alternative (vs the rejected al ternative), choice satisfaction is lower because its unique bad features will be especially apparent, and the unique bad features of the rejected alternative will be neglected.

The results of Houston et al. (1991) suggest that the way choice options are represented (e.g., via features) and the comparison process is conducted (e.g., which option is the focus of attention or how options are processed) may also play an important role in choice-process satisfaction. In this research we treat choice-process satisfaction as a dependent measure and examine how option limitation and option information-particularly the alignability and ease of comparison of the information-affect satisfaction with the choice process. We show that option limitation (i.e., making one option unavailable from a set of equally attractive options) decreases choice-process satisfaction, whereas the comparability of choice options through alignable features increases satisfaction. These two factors also interact to influence the choice-process satisfaction. Decreases in satisfaction due to option limitation, relative to all options being available, occur for people who are given a set of options in which the difference features are alignable (i.e., differences of a corresponding dimension; see Markman \& Gentner, 1993) but not for people who are given a set of options in which the difference features are nonalignable (i.e., differences of unique dimensions). We propose that alignable differences are easier to compare and carry more weight in people's attribute processing, and thus give rise to a perception of a greater amount of information about the option set that is relevant for choice. Making an option unavailable in this case would have a bigger impact than in a situation in which all options have nonalignable differences. Nonalignable differences are difficult to process and are likely to make people less aware that there is much information about the options that is relevant for decision making. When people are less aware that there is much relevant information, they will be less capable of knowing the value of the available options as well as that of the limited option. As a result, people are likely to be less satisfied overall, and whether or not a limitation occurs will 
not make much of a difference. In the following sections, we briefly review the relevant research on option limitation and comparison-based decision making. We then present four experiments to demonstrate the main effects of feature alignability and option limitation, their interaction on choice-process satisfaction (Experiments 1 and 3), and the underlying determinants of these effects, such as ease of comparison and perceived amount of option information (Experiments 2 and 3). Finally (in Experiment 4), we show that processes conducive to feature processing and an awareness of the amount of information about the options (i.e., increasing people's processing ability through consideration set formation) will enhance choice-process satisfaction even when features of the options are not easy to compare.

\section{Option Limitation}

The phenomenon of option limitation has been only indirectly dealt with in the choice literature. Farquhar and Pratkanis (1987) and Pratkanis and Farquhar (1992), for example, investigatetheimpact of "phantom" alternatives, i.e., unavailable options, on choice probabilities. While traditional models of individual choice assume that the addition of an unavailable alternative to a choice set will have no impact on the ratios of choice probabilities among the original alternatives (Luce, 1959), F arquhar and Pratkanis demonstrate cases in which the assumption is violated. They show that an unavailable alternative has potential contrast and attribute importance effects and thus influences the choice probabilities of the original alternatives. While this research suggests that the unavailability of an option can affect preference for options in the set, our interest is in how unavailability of an option may affect the decision maker's experience of making a choice.

In his theory of psychol ogical reactance, Brehm (1966) suggests that decision makers' reaction to a limitation of a choice option will vary in proportion to the importance of the option. For example, reaction to the limitation of a choice option is greater as preference for the alternative, as well as the importance of the alternative, increases (e.g., an automobile vs a toaster). Though important, Brehm's suggestion does not directly deal with decision makers' reaction toward the experience of having a choice option limited while holding desirability of the choice options constant. In order to assess choice-process satisfaction, we create a set of equally attractive choice options and test the presence and absence of option limitation on decision makers' satisfaction with the choice experience. Under these conditions, weexpect that people's choice-process satisfaction will be greater when all the options are available versus when an option is limited.

Underlying the above-mentioned studies of unavailable options is the important assumption that people process attribute information for each option, which enables them to assess the options and to be influenced by the unavailability of a choice option. Next we discuss how representation of sets of choice options may influence the processing of options and choice-process satisfaction. 


\section{Comparison and Feature Alignability}

Comparison processes play a fundamental role in judgment and choice. Research on attribute-based evaluation (Sanbonmatsu, Kardes, \& Gibson, 1991) and on similarity and comparison (Markman \& Medin, 1995) suggests that processing of options favor comparable features over noncomparable features, also known as alignable differences and nonalignable differences (see Gentner, 1983, 1989; Markman \& Gentner 1993, for comparison process as an alignment of hierarchical relational structures).

A comparison of two items, $X$ and $Y$, is not just a straightforward matter of comparing all of the attributes or features of $X$ with all of those of $Y$ (Tversky, 1977). Studies have shown that features that are unique to the comparing item (e.g., $X$ ) tend to be highlighted over the unique features of the item being compared to (e.g., Y): the so-called direction-of-comparison effect (e.g., H ouston, Sherman, \& Baker, 1989, 1991; Tversky, 1977; Tversky \& Gati, 1982). Of particular importance was the finding that directional effects in preference judgments depend on the nature of the features involved in the comparison process (Sanbonmatsu, Kardes, \& Gibson, 1991). This effect was observed when the features of two options were from different dimensions-unique or nonalignable (e.g., "Iow level of sodium" vs "large-sized corn") - but not when the features occupied different points on the same dimension-features that are alignable (e.g., "large-sized corn" vs "medium-sized corn"). This suggests that feature-based comparisons may be characterized by a search of the items under comparison for alignable features rather than for nonalignable features. In other words, the kinds of features of which options are composed may affect feature-based processing and the perceived information of the options.

Significant evidence exists for the distinction between alignable and nonalignable differences in comparisons and their differential effects in cognitive tasks. In particular, alignable differences are given more weight in similarity comparisons than are nonalignable differences, they are listed more often than arenonalignabledifferences, and they are morelikely to appear as justifications for decisions than are nonalignable differences (Gentner \& Markman, 1994; Markman \& Gentner, 1996; Markman \& Medin, 1995). More importantly, choice options represented by alignable differences vs nonalignable differences can lead to significantly different patterns of preference judgments for the options. In Zhang and Markman (1998), alignable differences of the choice options are recalled better than nonalignable differences, and alignable differences rather than nonalignble differences serve as input for preference construction.

With respect to choice-process satisfaction, we suggest that alignable differences, relative to nonalignable differences, facilitate the comparison process and choice-process experience. By focusing on alignable differences, the alignment process ensures that all choice options have a comparable representation. Further, alignable differences are easier to process and evaluate than nonalignable differences. These properties are likely to increase people's satisfac- 
tion experience when they choosefrom a set of options with alignabledifferences rather than nonalignable differences.

Moreover, the comparability or ease of comparison of alignable differences may allow them to receive more attention and elaboration. During comparison of choice options, participants are found to attend more to features that are alignable rather than nonalignable (e.g., Slovic \& MacPhillamy, 1974). Thus, alignable differences are likely to be processed more completely than nonalignabledifferences. The comparability of alignable features may al so enhance the perceived amount of information of options and the relevance of alignable features for decision making, because differences on a common dimension serve as better judgment cues than do nonalignable features. Because of these reasons, people are likely to feel less satisfied with the choice process when they experience a psychological loss of perceived relevant information through a choice option limitation relativeto a situation in which all options areavailable. In contrast, nonalignable features are harder to process and may suggest omission of information (Sanbonmatsu, Kardes, \& Herr, 1992; Sanbomatsu, Kardes, Posavac, \& Houghton, 1997). The presence of features in one item and the absence of the same features in the other item may be perceived as offering a smaller amount of the total possible information of the options. Thus, nonalignable differences are likely to be processed less completely and to be perceived as less relevant for decision making in comparison to alignable differences. When people are not aware that there is much relevant information, they may not feel capable of judging the options. In this situation, choiceoptions represented by nonalignable differences will make people feel less satisfied with the decision-making process regardless of choice limitation situation.

In sum, we predict that choice-process satisfaction will be greater when options are represented by alignable features than when they are represented by nonalignable features, and when all options are available than when an option is limited. Moreover, we predict that choice-process satisfaction will depend upon an interaction between the types of difference features used to represent the options in the choice set and the presence of an option limitation. For alignable-difference options, satisfaction should be less when one of the options is limited rather than when all options are available. For nonalignabledifference options, satisfaction should not differ significantly when one of the options is limited or when all the options are available. N ext, we present four experiments to test these hypotheses.

\section{EXPERIMENT 1}

Method

Subjects. One hundred eighty-seven undergraduate students from the UCLA community participated in the study in exchange for a payment of $\$ 5$. Participants reported to an auditorium and were assigned randomly to conditions by receiving different booklets. 
Stimuli. Attribute descriptions are shown in Table 1. Two sets of three brands were constructed. All brands had nine attributes. Four of these attributes were commonalities, while the remaining five attributes weredifferences. One set of three brands had alignable differences (we refer to this as the alignable condition), while the other set of three brands had nonalignable differences (we refer to this as the nonalignable condition). One brand, Brand $C$ in Table 1, was identical across the two alignability conditions. Attributes chosen for each type of feature difference (i.e., commonalities, alignable differences, and nonalignable differences) were randomly drawn from a list of attributes.

Before the main study, an independent pool of 46 subjects took part in a pretest to provide attribute ratings. The attributes were rated on a semantic differential scale with endpoints 1 for "extremely bad" and 9 for "extremely good." Attributes of the brands were designed such that the three brands in each condition were approximately equal in terms of desirability. Ratings in each condition were as follows: $A=6.02, B=6.05, C=6.12, F<1$ in the alignable condition, and $A=5.93, B=5.89, C=6.12, F<2$ in the nonalignable condition.

Design. The design is a 2 (Difference Type: alignable difference, nonalignable difference) $\times 2$ (Availability: all options available, one option not available) between-subjects design. Half of the participants saw the brands in the alignable set; the other half saw the brands in the nonalignable set. The three brands were presented on the same sheet of paper, with order of brands from left to right counterbalanced between subjects.

\section{TABLE 1}

Individual Attributes for Brands in Experiment 1

\begin{tabular}{|c|c|c|c|}
\hline & Brand A & Brand B & Brand C \\
\hline \multirow{5}{*}{$\begin{array}{l}\text { Alignable } \\
\text { differences }\end{array}$} & Uses Southwest corn & Uses Midwest corn & Uses Northwest corn \\
\hline & Medium-size kernels & Good-size kernels & Large kernels \\
\hline & $\begin{array}{l}\text { Comes with microwave } \\
\text { bowl }\end{array}$ & $\begin{array}{l}\text { Comes with disposable } \\
\text { microwave bags }\end{array}$ & Pops in its own bag \\
\hline & $\begin{array}{l}\text { Calories equal to a } \\
\text { tablespoon of sugar }\end{array}$ & $\begin{array}{l}\text { Calories equal to } 20 \\
\text { peanuts }\end{array}$ & $\begin{array}{l}\text { Calories equal to a slice } \\
\text { of bread }\end{array}$ \\
\hline & Crunchiness lasts long & $\begin{array}{l}\text { Crunchiness lasts for } \\
\text { three hours }\end{array}$ & $\begin{array}{l}\text { Crunchiness lasts within } \\
\text { a specified time }\end{array}$ \\
\hline \multirow{7}{*}{$\begin{array}{c}\text { Nonalignable } \\
\text { differences }\end{array}$} & Kind of crispy & Few kernels left & Uses Northwest corn \\
\hline & Not likely to burn & unpopped & Large kernels \\
\hline & Tastes a bit sweet & Some oil & Pops in its own bag \\
\hline & Easy to swallow & Comes in one package & Calories equal to a slice \\
\hline & Low in corn, grain & size & of bread \\
\hline & flavor & Not tough & Crunchiness lasts within \\
\hline & & Has fiber & a specified time \\
\hline
\end{tabular}

Note Commonalities are constant for all brands: fairly low level of sodium, easy to prepare, easy to store, and fairly low cost per servings. One fixed order of randomly mixed attributes was used for all brands. 
Procedure Each participant received a booklet and was told to follow the instructions closely. Instructions on the front page explained that this was a grocery study about choosing brands of microwave popcorn. Participants were told to imagine that they were interested in purchasing microwave popcorn and that they were standing in a supermarket aisle and examining brands of popcorn. On the following page, they received attribute descriptions about three brands-Brand A, Brand B, and Brand C, either the alignable set or the nonalignable set. For half of the participants, one of the three brands (Brand C) had a "tag" pasted over it which read "Sold Out." F or the other half of the participants, all three brands were available. Participants were asked, of the brands available to them, which would they choose. On the following page, partici pants completed a series of questions designed to measure their satisfaction with the decision process (Fitzsimons, Greenleaf, \& Lehmann, 1997). These items were as follows (all items have endpoints $1=$ strongly agree, $10=$ strongly disagree, unless otherwise noted): (1) I found the process of deciding which product to buy frustrating; (2) Several good options were available for me to choose between; (3) How satisfied or dissatisfied are you with your experience of deciding which product option to choose? (endpoints 1 = extremely satisfied, 10 = extremely dissatisfied); (4) I thought the choice selection was good; (5) I would be happy to choose from the same set of product options on my next purchaseoccasion; and (6) I found the process of deciding which product to buy interesting. Finally, participants were debriefed.

\section{Results}

A choice-process satisfaction index was constructed by averaging the six items (Cronbach's $\alpha=0.82$ ). In addition to the reported six-item composite measure, we also computed a three-item general satisfaction measure (Items 1,3 , and 6) and found no substantial differences between the full six-item and the three-item composite measures. Thus we report only the six-item measure. The index range was from 1 to 10, with higher numbers indicating greater levels of satisfaction. The mean levels of satisfaction ranged across conditions from a high of 6.4 to a low of 5.2 and are shown in Fig. 1. The choice share data were almost equally distributed among the brands in each condition, conforming to our stimuli set-up that each brand should be equally attractive (nonalignable/C unavailable: $\mathrm{A}=49 \%, \mathrm{~B}=51 \%$; nonalignable/available: $\mathrm{A}=$ $33.3 \%, B=31.1 \%, C=35.6 \%$; alignable/C unavailable: $A=53.3 \%, B=46.7 \%$; alignable/available $\mathrm{A}=34.8 \%, \mathrm{~B}=28.3 \%, \mathrm{C}=37 \%$ ). The fact that brand $\mathrm{C}$ was not chosen more often than the other brands in the available conditions suggests that the differential effect of satisfaction between alignability conditions in Fig. 1 is not contingent upon the choice of brand C.

A $2 \times 2$ between-subjects ANOVA was performed on the choice-process satisfaction scores with factors (i) Difference Type and (ii) Availability. The omnibus model was significant, $F(3,183)=6.48, p<.01$, as were the main effects of availability $F(1,183)=5.73, p<.05$, and difference type, $F(1,183)=9.32$, $p<.01$. The main effect of availability showed significantly lower levels of 


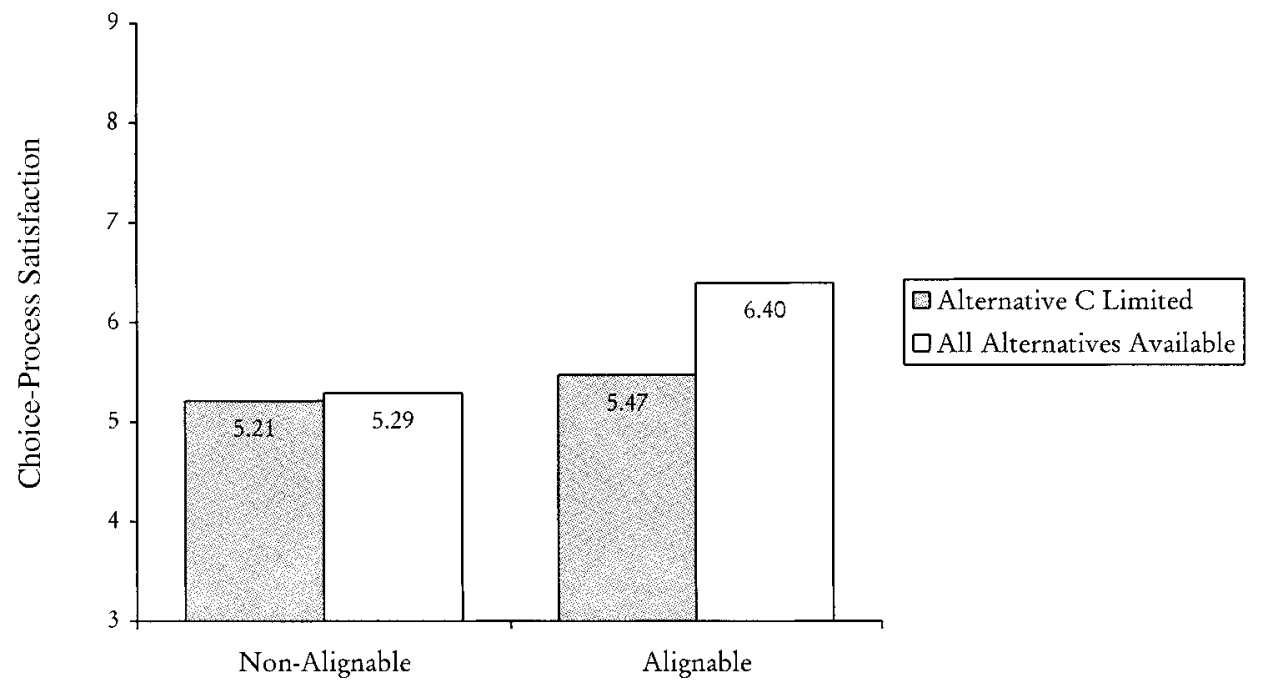

Attribute Alignability

FIG. 1. Choice-process satisfaction as a function of attribute alignability and option limitation.

satisfaction for participants who had their choice sets restricted by receiving an unavailable option. The main effect of difference type demonstrated significantly higher levels of satisfaction for participants who chose from sets that had alignable differences (venus nonalignable attributes). The analysis also revealed a two-way interaction between availability and difference type, $F(1$, $183)=4.19, \mathrm{p}<.05$. As predicted, a planned contrast between conditions showed that there was a significant decrease in choice-process satisfaction due to the presence of an unavailable option for the alignable condition but not for the nonalignable condition. In the alignable condition, mean satisfaction decreased from 6.40 to 5.47 due to limitation, $F(1,183)=9.85, p<.01$. In the nonalignable condition, satisfaction ratings were approximately equal between those who received an unavailable option and those who did not, $M=5.21$ versus $M=5.29, F(1,183)<1$. Finally, satisfaction was significantly higher in the alignable condition than in the nonalignable condition when all options were available $(M=6.40$ vs $M=5.29), F(1,183)=12.80, p<.01$.

\section{Discussion}

The findings conform to our expectations. First, people like the availability of options in the choice set. This is intuitive and easy to understand. Second, partici pants who are given alignable difference options show more satisfaction than those who are given the nonalignable difference options. Alignable differences, as suggested earlier, often serve as heuristic dimensions for decision making and are easier to process, relative to nonalignable differences. As a result, processing alignable-difference options is a relatively more pleasant experience. The interesting result is that participants' satisfaction with the choice process was significantly lower when an option was limited than when 
all options were available for the choice set of alignable-difference options; however, this contrast is absent for the nonalignable-difference options (i.e., satisfaction is low regardless of option limitation).

We have suggested that alignable difference options would allow participants to engage in easier comparison and more complete processing of option information and to have a perception of more attribute information available and rel evant for choice than do nonalignable difference options. It was our assumption that when people (in the alignable condition) feel an ease of comparison they will have a pleasant experience during the choice process. In addition, when people (in the alignable condition) are more aware that they are processing information perceived to be relevant for decision making and are capable of judging options, losing one of the options will make them feel less satisfied about the decision process than people (in the nonalignable condition) who are less aware that there is much information relevant for choice to begin with. However, whether people indeed feel the ease of comparison and perceive more attribute information about the options needs to be empirically verified. Experiment 2 was conducted to examine whether, in a decision-making context, participants had the subjective experience that the comparison process was easier and that they were attending to more information of options for choice when they were processing alignable differences than when they were processing nonalignable differences. Experiment 3 varies the ease of comparison to increase the ability to process attributeinformation. It replicates Experiment 1 and demonstrates that enhanced ease of comparison leads people to feel more satisfied about their choice process relative to a baseline ease of comparison as well as relative to a reduced ease of comparison.

\section{EXPERIMENT 2}

Method

Thirty-six students from a class at UCLA were shown the same sets of options as in Experiment 1, the alignable difference option set or the nonalignable difference option set (without being given the option limitation information). The participants were told to consider making a choice from one of the option sets and then to provide responses to the dependent measures for ease of comparison and perceived amount of information of options. Ease of comparison was measured on a 9-point scale: In general, you find that comparing these brands and their attribute description seems (not at all easy-very easy). Perceived amount of information was measured on two 9-point scales: In general, you find that the amount of information provided about each brand for decision making seems (a little-a lot); in general, you find that the information provided about each brand seems (not at all complete-very complete).

\section{Results and Discussion}

The results are shown in Fig. 2. A t test performed on the ratings of ease of comparison revealed a significant difference between the groups, $\mathrm{t}(34)=3.49$, 

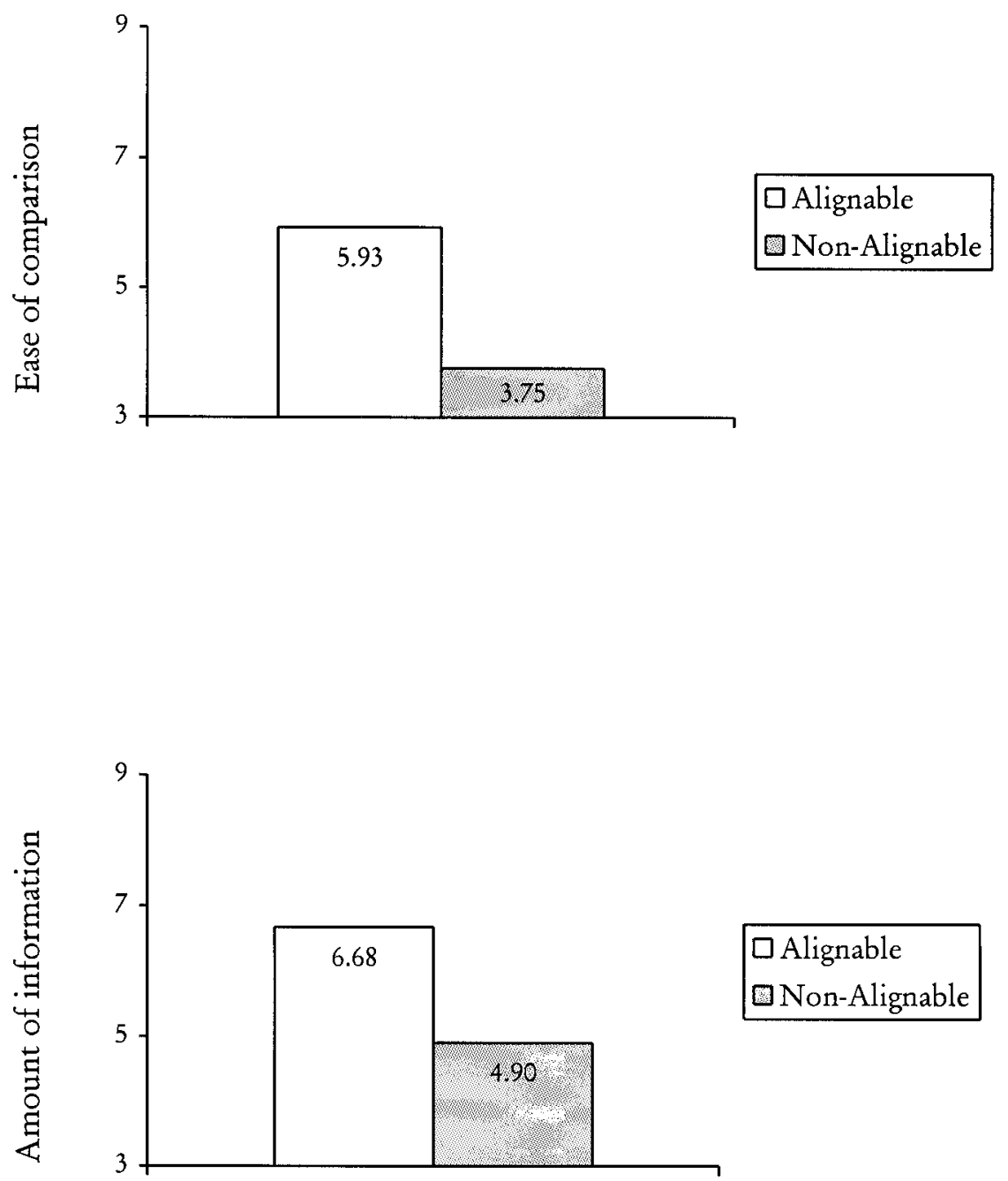

$\square$ Alignable
Non-Alignable

FIG. 2. Ease of comparison (top) and perceived amount of information (bottom) as a function of attribute alignablility.

$p<.01$. Participants presented with alignable-difference options perceived it to be easier to compare the attribute descriptions than did participants who were presented with nonalignable-difference options ( $M=5.93$ vs $M=3.75$ ). Moreover, a t test also revealed a significant effect of alignability on the perceived amount of information about the options (an index averaging the two items about information is used, corr $=0.62, p<.001$ ). Participants in the alignable difference condition perceived significantly more information relevant for choice than did those in the nonalignable difference condition ( $M=$ 6.68 vs $M=4.90), t(34)=3.46, p<.01$. Our contention is that alignable difference options are easier to compare and are perceived to offer more option information than nonalignable difference options (corr (ease of comparison, amount of information) $=0.41, p<.05$ ). The results of this experiment support this contention. 
In our stimuli construction (which uses nine attributes for each option), ease of comparison can be thought of as a combination of feature alignablity and commonalties for choice options. For example, with the total number of attributes as well as the number of commonalities kept constant, theuse of alignable (vs nonalignable) differences should result in greater ease of comparison. In addition, when the number of alignable differences is held constant, an increase in the number of commonalties should result in easier comparison (see Gentner \& Markman, 1994, on relation between commonalities and similarity). Earlier we reasoned that ease of comparison increases the ability to process attributeinformation. Assuming that ease of comparison al so facilitates participants to perceive that there is a greater amount of information available for choice, we expect participants to experience an overall higher choice-process satisfaction when the level of ease of comparison is enhanced. U pon the removal of a choice option, i.e., information, partici pants' level of choice-process satisfaction should significantly decrease relative to no option limitation.

In the next experiment, we manipulate the level of ease of comparison. Relative to a set of baseline options that have three commonalities and six alignable differences, we enhance the level of ease of comparison by increasing the number of commonalities and simultaneously reducing the number of alignable differences-six commonalities and three alignable differences. Conversely, relative to the same baseline options, we decrease the level of ease of comparison by keeping the same number of commonalities but substituting nonalignable differences-three commonalities and six nonalignable differences. The predictions are as follows: (1) The overall satisfaction with the decision process should be higher for the participants in the enhanced-easeof-comparison condition than in the baseline and reduced-ease conditions, and (2) participants should feel less satisfied facing an option limitation relative to no option limitation, in both the baseline and enhanced-ease-of-comparison conditions (i.e., the alignable-differences conditions); however, there should be no such difference in the reduced-ease-of-comparison condition (i.e., the nonalignable-differences condition).

\section{EXPERIMENT 3}

\section{Method}

Subjects. One hundred five undergraduate students from the UCLA community took part in this experiment in exchange for a payment of $\$ 5$. They cameto an auditorium and were randomly assigned to experimental conditions.

Stimuli. Three sets of three fictitious brands each were constructed, the reduced-ease-of-comparison nonalignable set (henceforth reduced-ease), the baseline-ease-of-comparison alignable set (henceforth, baseline-ease), and the enhanced-ease-of-comparison alignable set (henceforth enhanced-ease), as shown in Table $2 \mathrm{~A}$ and $2 \mathrm{~B}$. Each brand had nine attributes in total. The reduced-ease and baseline-ease sets had brands that were constructed with 
TABLE 2A

\section{Added Individual Difference Attributes for Brands with Three Commonalities and Six Differences in Experiment 3}

\begin{tabular}{lccc}
\hline & Brand A & Brand B & Brand C \\
\hline Alignable differences & Can be stored for & Can be stored for & Can be stored for \\
Nonalignable differences & 5 months & 6 months & 7 months \\
& Has 0.5 g of protein & $\begin{array}{c}\text { Has } 1 \% \text { of daily } \\
\text { vitamin C }\end{array}$ & $\begin{array}{c}\text { Can be stored for } \\
7 \text { months }\end{array}$ \\
\hline
\end{tabular}

Note Commonalities are constant for all brands: easy to prepare, easy to store, and fairly low cost per serving. The other five differences, alignable or nonalignable, for each brand are the same as in Table 1. One fixed order of randomly mixed-up attributes was used for all brands.

TABLE 2B

Individual Attributes for Brands with Six Commonalities and Three Differences in Experiment 3

\begin{tabular}{clll}
\hline & \multicolumn{1}{c}{ Brand A } & Brand B & \multicolumn{1}{c}{ Brand C } \\
\hline $\begin{array}{c}\text { Alignable } \\
\text { differences }\end{array}$ & $\begin{array}{c}\text { Medium-size kernels } \\
\text { Comes with microwave } \\
\text { bowl } \\
\text { Calories equal to a } \\
\text { tablespoon of sugar }\end{array}$ & $\begin{array}{c}\text { Good-size kernels } \\
\text { Comes with disposable } \\
\text { microwave bags } \\
\text { Calories equal to 20 } \\
\text { peanuts }\end{array}$ & $\begin{array}{c}\text { Large kernels } \\
\text { Pops in its own bag } \\
\text { Calories equal to a slice } \\
\text { of bread }\end{array}$ \\
\hline
\end{tabular}

Note Commonalities are constant for all brands: fairly low level of sodium, easy to prepare, easy to store, fairly low cost per serving, $0.5 \mathrm{~g}$ of protein, and, waterproof wrapping. One fixed order of randomly mixed attributes was used for all brands.

three commonalities and six differences (nonalignable differences for the reduced-ease and alignable differences for the baseline-ease). The enhanced-ease set had brands with six commonalities and three alignable differences.

Pretesting confirmed that the three brands in each of the three sets were relatively equal in terms of brand desirability: the reduced-ease nonalignable set $(A=6.58, B=6.58, C=6.70), F<1$; the baseline-ease alignable set $(A=6.96, B=7.0, C=6.90), F<1$; and the enhanced-ease alignable set $(A=6.01, B=6.10, C=6.34), F<1$.

Design. The experimental design took the form of a between-subjects partial factorial design, 2 (Difference Type: alignable difference, nonalignable difference) $\times 2$ (Availability: all options available, one option not available) $\times 2$ (Number of Commonalities: three commonalities, six commonalities). The first two factors are exactly the same as those in Experiment 1 and yield four conditions that contain options with three commonalities: reduced-ease nonalignable/one option unavailable or all options available, baseline-ease alignable/one option unavailable or all options available. The third factor generates the enhanced-ease set conditions that have six commonalities in option descriptions: enhanced-ease/one option unavailable or all options available. There are six conditions in total. 
Procedure The procedurefollowed was identical to that used in Experiment 1. Participants received the same instructions, were exposed to the stimulus brands, made a choice, and then answered the choice-process satisfaction questions. Half of the participants had all of the options available for choice, while the remaining half of the sample had a "tag" which read "Sold Out" pasted over the heading of Brand $\mathrm{C}$.

\section{Results}

As in Experiment 1, a choice-process satisfaction index was created by averaging the items (Cronbach's $\alpha=0.79$ ). Mean satisfaction ratings across the six conditions ranged from a low of 4.92 to a high of 7.04. See Fig. 3 for a summary of the cell means. Choice share data were almost equally distributed among the brands for each condition, similar to those in Experiment 1 (nonalignable/C unavailable: $A=50 \%, B=50 \%$; nonalignable/all options available: $\mathrm{A}=38.9 \%, \mathrm{~B}=22.2 \%, \mathrm{C}=38.9 \%$; alignable/C unavailable: $\mathrm{A}=47.1 \%, \mathrm{~B}=$ 52.9\%; alignable/all options available: $\mathrm{A}=33.3 \%, \mathrm{~B}=33.3 \%, \mathrm{C}=33.3 \%$; enhanced alignable/C unavailable: $A=48.89, B=51.1 \%$; enhanced alignable/ all options available: $A=27.65 \%, B=35.2 \%, C=36.2 \%)$.

A $2 \times 2 \times 2$ partial factorial analysis of variance (ANOVA) was conducted on levels of choice-process satisfaction for participants (Box \& Hunter, 1978). The overall model was significant, $F(5,99)=3.83, p<.01$, as was the main effect of availability, $F(1,99)=6.62, p<.001$, and the main effect of number of commonalities, $F=3.74, p<.05$. The main effect of availability suggests that the presence of an unavailable brand led to lower levels of satisfaction than when all options wereavailable. Themain effect of the number of commonalities suggests that participants who were exposed to the enhanced-ease alignable set had overall higher levels of satisfaction than those who were given the baseline-ease alignable set and reduced-ease nonalignable set. This finding is

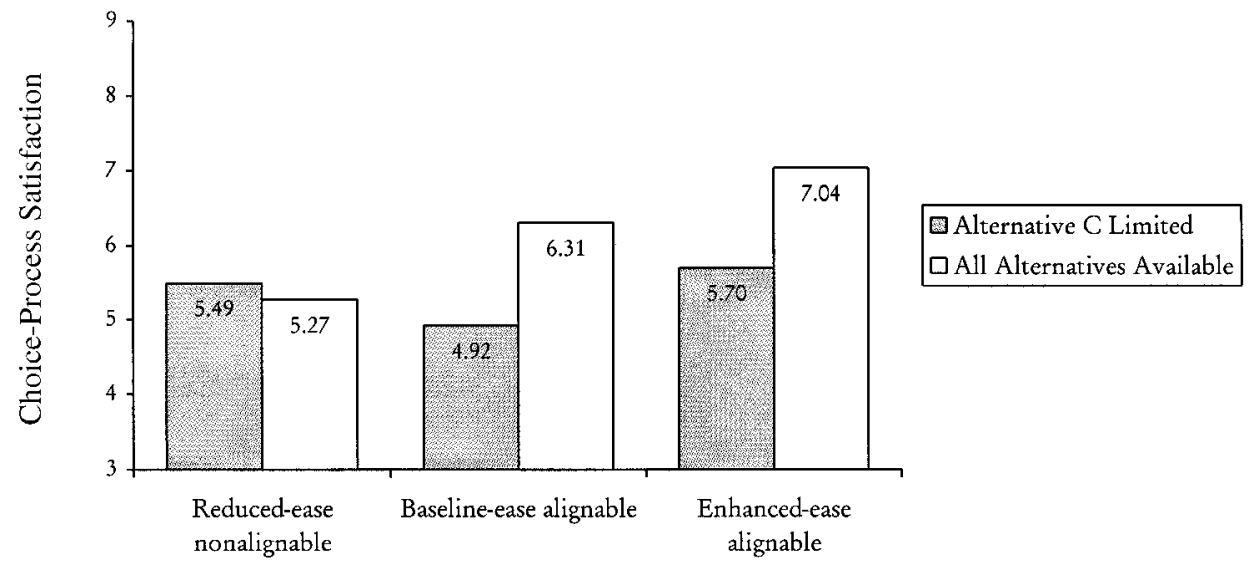

FIG. 3. Choice-process satisfaction as a function of attribute alignability, option limitation, and ease of comparison. Common to each set of options in reduced-ease and baseline-ease are three commonalities and six differences. Common to each set of options in enhanced-ease are six commonalities and three differences. 
important, as it confirms our prediction that enhancing ease of comparison should increase overall level of satisfaction with the choice process.

Furthermore, the two-way interaction between availability and difference type was significant, $F(1,99)=4.21, p<.05$, replicating the results of Experiment 1 . Under both the baseline-ease alignable and enhanced-ease alignable conditions, a significant decrease in participants' choice-process satisfaction due to "Brand C unavailable" was observed relative to "all options available": $M=4.92$ vs $6.31, F(1,99)=3.63, p=.05$, and $M=5.70$ vs $M=7.04, F(1$, $99)=5.29, p<.05$. However, under the reduced-ease nonalignable set condition, as expected, no such difference in satisfaction was observed for those who were given an unavailable option ( $M=5.49$ ) versus those who were not $(M=$ 5.27), $\mathrm{F}<1$. Consistent with our predictions, these results clearly show that feature alignability moderates people's choice-process satisfaction when an option from an equally attractive choice set was limited.

A separate follow-up $2 \times 2$ analysis of variance was conducted to further corroborate the findings. For participants in the reduced-ease nonalignableand the baseline-ease alignable conditions, the 2 (difference type) $\times 2$ (availability) model yiel ded a significant interaction effect between difference type and availability, $F(1,66)=3.97, p<.05$, similar to the result obtained in Experiment 1. Pooling across the baseline-ease alignable condition and the enhanced-ease alignable condition, the 2 (number of commonalities) $\times 2$ (availability) model revealed a significant main effect of availability, $F(1,67)=11.76, p<.001$, as expected. More important, the model also revealed a significant main effect of number of commonalities, $F(1,67)=3.67, p=.05$. As we predicted, participants expressed higher levels of satisfaction in the enhanced-ease condition than in the baseline-ease condition. No other effects were significant.

\section{Discussion}

In this experiment, we observed a contrast between all options available vs option limitation for the baseline-ease condition as well as the enhanced-ease condition but not the reduced-ease condition. More important, we observed the expected overall increase of satisfaction in the enhanced-ease condition relative to the baseline-ease condition. These results suggest that ease of comparison via alignable differences (between the alignable and nonalignable sets) as well as via commonalities (between the baseline-ease alignable and enhanced-ease alignable sets) facilitates feature processing and increases satisfaction with the decision process.

The contrast between all options available vs option limitation in the enhanced-ease condition was approximately the same as that in the baseline-ease condition, though one might expect a larger contrast for the former condition because of the enhancement in the ease of comparison. It seems reasonable to suggest that the magnitude of any decrease in satisfaction due to option limitation is dependent upon ease of comparison as well as perceived amount of information. Perhaps the ease of comparison through commonalities does not necessarily contribute as much perceived option information as does the ease 
of comparison through alignable differences. Thus, the contrast between option availability vs option limitation for our enhanced-ease conditions was not larger than for the baseline-ease condition.

Experiments 2 and 3 together show that participants have the subjective experience that they are attending to a greater amount of information relevant for decision making in the alignable condition than in the nonalignable condition. When people (in the alignable condition) are more aware that they are processing a greater amount of information that appears relevant for decision making, they may feel that they are capable of judging the options. Thus, having one of the options limited makes them feel less satisfied about the decision than people (in the nonalignable condition) who are less aware that there is much decision-relevant information to begin with for judgment. As suggested earlier, processing of nonalignable differences is likely to be less complete, because they are difficult to process and are less attended to, relative to alignable differences.

Our results and analysis suggest that featurealignability and ease of comparison facilitate attribute information processing, which makes people more aware of the amount of attribute information available of all options. In particular, we have shown that nonalignable differences are not conducive to the processing of or the awareness of attribute information of options. According to this view, when participants' ability to process nonalignable differences is increased, they could be made more aware of the information of all options. As a result, we should observe a similar pattern of choice-process satisfaction to that found with alignable-difference options. Experiment 4 is conducted to test this prediction.

\section{EXPERIMENT 4}

In Experiment 4, we use the same set of nonalignable-difference options as in Experiment 1 plus two additional nonalignable difference options, as shown in Table 3. We manipulate the level of ability to process the information by requiring (vs not requiring) the participants to first pick three options out of

TABLE 3

Individual Attributes for Added Brands in Experiment 4

\begin{tabular}{|c|c|c|}
\hline & Brand D & Brand $\mathrm{E}$ \\
\hline \multirow[t]{5}{*}{ Nonalignable differences } & Has $0.5 \mathrm{~g}$ of protein & Has $1 \%$ of daily vitamin C \\
\hline & Very salty & Has some citric acid \\
\hline & Yellow corn & Very filling \\
\hline & $\begin{array}{l}\text { Box made with } 1 \% \text { recyclable } \\
\text { paper }\end{array}$ & With waterproof wrapping \\
\hline & Takes 7 min to pop & $\begin{array}{l}\text { Includes a very spicy topping } \\
\text { package }\end{array}$ \\
\hline
\end{tabular}

Note Attributes of Brands A, B, and C are referred to in Table 1. Commonalities are constant for all brands, same as in Table 1. 
a set of five. The five options are constructed based on a pretest such that after a fair amount of relatively intensive comparison and processing participants will be able to select brands $A, B$, and $C$ and eliminate brands $D$ and $E$. We assume that this consideration formation process will have forced the participants to process and use nonalignable differences for decision making. After the consideration set is formed, participants will be exposed to the unavailability of an option (vs all options are available) manipulation. We expect that the participants who are given the consideration formation manipulation should yield a higher level of choice-process satisfaction, and exhibit a significant decrease in satisfaction when a choice option is limited relative to when all options are available.

\section{Method}

Subjects. Fifty-eight undergraduate students from UCLA participated in exchange for a payment of $\$ 5$. As before, they reported to a classroom and were randomly assigned to the conditions.

Stimuli. Five brands were constructed for this experiment. All five brands had nine attributes (four commonalities and five nonalignable differences). Three of the five brands were identical to those nonalignable-difference options used in Experiment 1. The two additional brands had nonalignable differences that were judged to be less desirable than those of the other three brands. The relationship in terms of desirability among the five brands was designed to match the pattern of $A=B=C<D=E(A=5.93, B=5.89, C=6.12, D=$ $4.95, E=4.80$, on a 9-point scale). Attribute descriptions are provided in Table 3. This pattern was chosen because we wanted participants to form a consideration set of $A, B$, and $C$ and make their selections from the set of the three original equally liked brands as in Experiment 1. Again, presentation of the brands from left to right was counterbalanced between subjects.

Design. Experiment 4 follows a 2 (Availability: all options available, one option not available) $\times 2$ (Consideration Set Formation: required, not required) between-subjects design.

Procedure The procedure followed was similar to that followed in Experiments 1 and 3. Participants received the same instructions and were presented with the five brand stimuli. Half of the subjects were asked to examine the attribute descriptions and indicate which three of the five brands they would be most likely to consider choosing by circling the brands. The other half was told to examine the attribute descriptions and decide which brand they would consider choosing. On the next page, all participants were presented with a notice and an "availability table" (which indicated which brands were currently available by having the term "available" or "not available" beside the brand). Participants were then asked to make a choice of popcorn, and to answer a set of questions regarding their satisfaction with the decision process. 


\section{Results and Discussion}

As in previous experiments, a choice-process satisfaction index was created (Cronbach's $\alpha=0.82$ ). As shown in Fig. 4, the average satisfaction across conditions ranged from a low of 5.84 (for participants who received an unavailability announcement but were not asked to form and report a consideration set) to a high of 7.51 (for participants who were asked to form and report a consideration set and did not receive the unavailability announcement). All participants in the consideration set formation condition circled Brands A, B, and $C$ as their set. The choice share data for the brands in each condition did not show unusual outcomes that could contribute possible different satisfaction outcomes (Not-Required/C unavailable: $A=53.3 \%, B=46.7 \%$; Not-Required/ all options available: $\mathrm{A}=38.5 \%, \mathrm{~B}=23.1 \%, \mathrm{C}=38.5 \%$; Required/C unavailable: $A=46.7 \%, B=53.3 \%$; Required/all options available: $A=40 \%, B=26.7 \%$, $\mathrm{C}=33.3 \%$ ). Notethat participants included for analysis in thenonconsideration formation condition were those who picked one of $A, B$, or C as their choice (two participants who chose options $D$ and $E$ were not included in these analyses).

A $2 \times 2$ ANOVA was performed on the choice-process satisfaction scores, yielding a significant overall model, $F(3,54)=6.13, p<.01$, a main effect of availability, $F(1,54)=6.28, p<.05$, and a main effect of consideration set formation, $F(1,54)=7.84, p<.01$. The main effect of availability showed significantly lower levels of satisfaction for participants who had their choice sets restricted by receiving an unavailable option. The main effect of consideration set formation suggested that participants who were required to form a consideration set had significantly higher levels of choice-process satisfaction than did those not required to form a consideration set. These results confirmed our prediction.

Moreover, the two-way interaction was significant, $F(1,54)=4.27, p<.05$. As predicted, for the condition in which participants were not required to form a consideration set, wefound noreliable differencein choice-process satisfaction

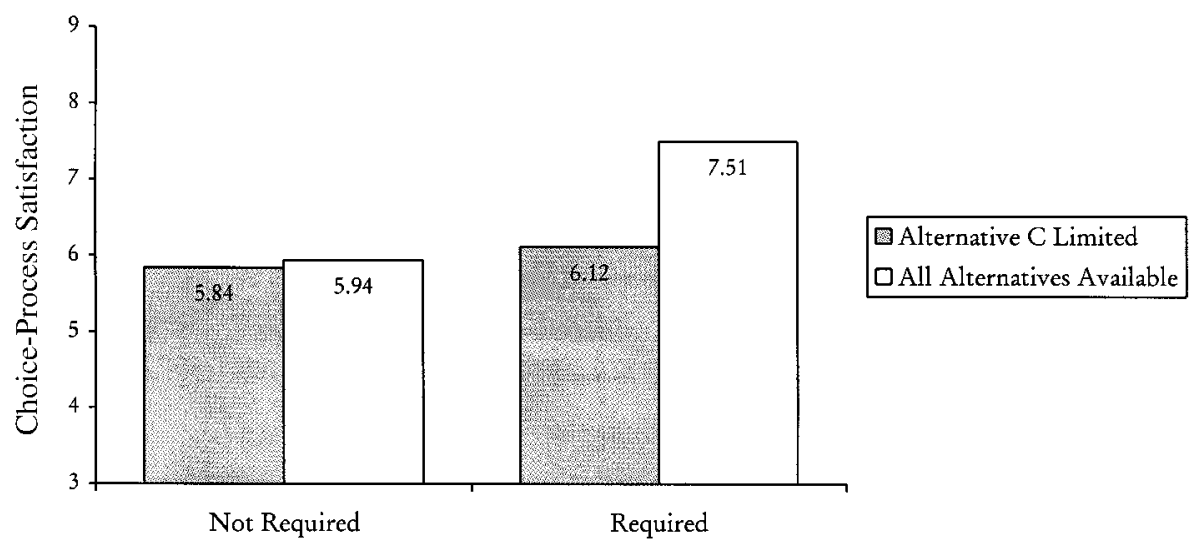

Consideration Set Formation

FIG. 4. Choice-process satisfaction as a function of consideration set formation and option limitation. 
between thosereceiving an unavailable option versus thosefor whomall options were available for choice $(M=5.87$ vs $M=5.94), F(1,54)<1$. This result replicated the findings of the previous experiments for the nonalignable difference set of options. More importantly, a planned contrast of conditions in which participants were required to form a consideration set found a significant difference in satisfaction. Participants who were shown an unavailable option had lower satisfaction ratings than those for whom all options were available $(M=6.12$ vs $M=7.51), F(1,54)=10.02, p<.01$.

These results clearly suggest that requiring participants to form and report a subset of options that they would consider choosing made the participants increase feature processing. The fact that all participants select $A, B$, and C, which are better options than $D$ and $E$, in their formed consideration set is a clear indication that a fair amount of processing and judgment has been performed on the attribute information. When people are made more aware of the information about the options and use the information in consideration set formation, having one of the considered brands limited will make them feel less satisfied about the decision process than if they have all options available for decision.

\section{GENERAL DISCUSSION}

The experiments were designed to examine the determinants of people's satisfaction with the choice process, particularly option information (feature alignability, ease of comparison, and perceived amount of information) and option limitation. Consistent with research on similarity and comparison (e.g., Houston et al., 1989, 1991; M edin, Goldstone, \& Markman, 1995; Sanbonmatsu et al., 1991), we hypothesized that the nature of the difference features may have differential impact on the perceived ease of attribute comparison and amount of information of options that is relevant for choice and therefore may make people have differential experience with the choice process when one of the equally attractive choice options becomes unavailable.

In Experiment 1, we found that participants' satisfaction with choice process was significantly higher when the equally attractive options were described by alignable differences than when they were described by nonalignable differences. Furthermore, when participants were exposed to a set of equally attractive options that had alignable differences, their satisfaction ratings decreased significantly from when all options were available to when one option was made unavailable. However, in choice sets that had nonalignable differences among the options, choice-process satisfaction was low overall regardless of whether an option was made unavailable or not.

As an explanation for the underlying process, in Experiment 2 we demonstrated that people were more likely to perceive greater ease of comparison and more information of options as relevant for choice when presented with alignable-difference options than when presented with nonalignable-difference options. Having some of that information psychologically unavailable for a decision would decrease people's satisfaction with the choice process relative 
to having all the options available, more for the alignable-difference options than for the nonalignable-difference options. Processing of nonalignable differences is likely to be less complete, because they are difficult to compare and process, and hence less attended to. Consequently, people perceive a lower amount of useful or relevant information for choice and may feel incapable of making judgments with the information. Making unavailable some of the information that is perceived to be not very useful versus not making it unavailable, therefore, does not make much of difference. Experiment 3 replicated the findings of Experiment 1 and further showed that enhanced ease of comparison through the use of fewer difference features and more common features increased the overall level of choice-process satisfaction.

To further corroborate the proposed process, we conducted Experiment 4 to present parallel results supporting the explanation that a process conducive to feature processing and awareness of amount of information of all options will enhance choice-process satisfaction. When participants' ability to process nonalignable-difference features is increased through being required to form a subset of options for consideration, participants expressed higher levels of satisfaction with the choice process. Moreover, these participants expressed less satisfaction with the process when an option became unavailable relative to when all options were available, whereas those whose processing ability was not increased exhibited no such difference in choice-process satisfaction.

Many decision-making environments involve comparison of options. Some of the options have alignable aspects of information, while others list only aspects that are unique to each option. The data from our studies suggest that participants' affective response toward the choice process is related to how the options are represented. Past research has examined the impact of prechoice conflict due to valence of features on satisfaction with the outcome (Houston et al., 1991), but not on satisfaction with the decision process per se. In an exploratory study of satisfaction with the decision process, Westbrook et al. (1978) identified a number of potential influences, one of the more important of which was breadth and availability of choice options. The current research focused specifically on participants' satisfaction with the process of decision making. Prior to this research, little was known about participants' affective reactions or feel ings toward the choice process in the context of featurecomparison and option limitation. Our results suggest that participants may have different subjective experiences with the ease of comparison and perceived amount of information rel evant for choice, depending on which type of difference features are present. Because of these differences, people's choice-process satisfaction is higher when options are described with alignable differences and processed easily and completely. These findings are an important addition to our knowledge that difference features that are comparable are, relative to noncomparable difference features, weighted more heavily in preferenceformation and used more often in justifications of decision-making behavior (Markman \& Medin, 1995; Slovic \& MacPhillamy, 1974; Zhang \& Markman, 1998).

Additionally, these findings suggest the potential for a broad model of the 
antecedents of choice-process satisfaction that perhaps includes not only sufficiency and quality of information that is expected by the decision maker (Spreng, MacKenzie, \& Olshavsky, 1996; Westbrook et al., 1978) and option limitation (Fitzsimons, 1998), but also perceived ease of making comparisons and the perceived amount of information relevant for choice. Perceived ease of comparison is manipulated mainly through feature alignability on multiple attribute dimensions. Although our studies indicate that ease of comparison facilitates perceived amount of information, the interaction between these variables can be complex. For example, an increase in ease of comparison may not be accompanied by a similar increase in the perceived amount of information if only two as opposed to six attribute dimensions are described. In the case of two, ease of comparison will be high, whereas the amount of information may be low. Future development of a model of decision-process satisfaction should examine these interactions in detail.

Finally, the present research may have implications for choice decision research. In choice studies, one typically examines participants' response to a pair of options that are both described on a set of common dimensions, not on a set of unique features (see exception of Houston et al., 1989). Take, for example, the subject of deferred decision making. People are more likely to defer decision when the choice between $X$ and $Y$ is difficult (e.g., when the alternatives are about equally attractive but not identical) than when the choice is easy (e.g., when $X$ dominates Y) (e.g., Tversky \& Shafir, 1992). As suggested by our results, choice may be even more difficult when $X$ and $Y$ are described by a set of uniquefeatures. In such a case, participants are morelikely to undergo a less satisfying experience when conducting feature comparison due to nonalignable differences of the alternatives in addition to decisions between alternatives that are relatively similar in attractiveness. Deferred decision should be more likely to happen, and people should be more resolute about the deferred decision, when choice options have nonalignable features rather than alignable features (Zhang \& Sood, 1999). When the choice-process results in satisfaction (through ease of comparison and perceived sufficient amount of information), it will provide an environment that is more likely to foster a decision-seeking attitude; on the other hand, unsatisfactory experience will evoke decision aversion (see Beattie, Baron, Hershey, \& Spranca, 1994, on decision attitudes).

In conclusion, people's experience and satisfaction with the choice process can be influenced by both information about the options in a choice set and option limitation. Information that is alignable (vs nonalignable), easier to process, and perceived to offer a greater amount of information for decision making increases satisfaction. Alignable information, in particular, facilitates ease of comparison and improves the subjective experience with the perception of more relevant information for decision making. Thus, having a choice option unavailable (vs all options available) has a bigger impact on people's satisfaction with the choice process for alignable choice sets than for nonalignable choice sets. 


\section{REFERENCES}

Andersen Consulting. (1996). Where to look for incremental sales gains: The retail problem of outof-stock merchandise Coca-Cola Retailing Research Council.

Brehm, J. W. (1966). A theory of psychological reactance. New York: Academic Press.

Beattie, J ., Baron, J., Hershey, J. C., \& Spranca, M. D. (1994). Psychological determinants of decision attitude. J ournal of Behavioral Decision Making, 7, 129-144.

Box, G. E. P., \& Hunter, W. G. (1978). Statistics for experimenters. New York: Wiley.

Farquhar, P. H., \& Pratkanis, A. R. (1987). Phantom choices: The effects of unavailablealternatives on decision making (Technical Report No. 87-2). Pittsburgh, PA: Carnegie Mellon University.

Festinger L. (1957). A theory of cognitive dissonance. Stanford, CA: Stanford Univ. Press.

Fitzsimons, G. J . (1998). Consumer response to stock-outs (Working Paper No. 258). Los Angeles: UCLA Marketing Studies Center.

Fitzsimons, G.J ., Greenleaf, E. A., \& Lehmann, D. R. (1997). Decision and consumption sati sfaction: Implications for channel relations (Working Paper No. 313). Los Angeles: UCLA Marketing Studies Center.

Gentner, D. (1983). Structure-mapping: A theoretical framework for analogy. Cognitive Science, 7, 155- 170.

Gentner, D. (1989). The mechanisms of analogical learning. In S. Vosniadou \& A. Ortony (Eds.), Similarity and analogical reasoning (pp. 199-241). New York: Cambridge Univ. Press.

Gentner, D., \& Markman, A. (1994). Structural alignment in comparison: No difference without similarity. Psychological Science, 5, 152-158.

Hess, J . D., \& Gersner, E. (1987). Loss leader pricing and rain check policy. Marketing Science, 6, 358- 374.

Houston, D. A., Sherman, S. J., \& Baker, S. M. (1989). The influence of unique features and direction of comparison on preferences. J ournal of Experimental Social Psychology, 25, 121-141.

Houston, D. A., Sherman, S. J ., \& Baker, S. M. (1991). Feature matching, unique features, and the dynamics of the choice process: Predecision conflict and postdecision satisfaction. J ournal of Experimental Social Psychology, 27, 411-430.

Luce, R. D. (1959). Individual choice behvior: A theoretical analysis. New York: Wiley.

Markman, A. B., \& Gentner, D. (1993). Splitting the differences: A structural alignment view of similarity. J ournal of Memory and Language, 32, 517-535.

Markman, A. B., \& Gentner, D. (1996). Commonalities and differences in similarity Comparisons. Memory and Cognition, 24, 235-249.

Markman, A. B., \& Medin, D. L. (1995). Similarity and alignment in choice. Organizational Behavior and Human Decision Processes, 63, 117-130.

Medin, D. L, Goldstone, R. L., \& Markman, A. B. (1995). Comparison and choice: Relations between Similarity processes and decision process. Psychonomic Bulletin and Review, 2, 1- 19.

Nowlis, S. M., \& Simonson, I. (1997). Attribute-Task compatibility as a determinant of consumer Preference reversals. J ournal of Marketing Research, 34, 205-218.

Pratkanis, A. R., \& Farquhar, P. H. (1992). A brief history of research on phantom alternatives: Evidence for seven empirical generalizations about phantoms. Basic and Applied Social Psychology, 13, 103-n122.

Sanbonmatsu, D. M., Kardes, F. R., \& Gibson, B. D. (1991). The role of attribute knowledge and overall evaluations in comparative judgment. Organizational Behavior and Human Decision Processes, 48, 131- 146.

Sanbonmatsu, D. M., Kardes, F. R., \& Herr, P. M. (1992). The role of prior knowledge and missing information in multi-attribute evaluation. Organizational Behavior Human Decision Processes, 51, 76-91. 
Sanbonmatsu, D. M., Kardes, F. R., Posavac, S. S., \& Houghton, D.C. (1997). Contextual influences on judgment based on limited information. Organizational Behavior and Human Decision Processes, 69, 251-264.

Sheppard, B. H., Lewicki, R. J ., and Minton, J . W. (1992). Organizational and justice: The search for fairness in the workplace New York: Macmillan.

Slovic, P., \& MacPhillamy, D. (1974). Dimensional commensurability and cueutilization in comparative judgment. Organizational Behavior and Human Performance, 11, 172-194.

Spreng, R. A., Mackenzie, S. B., \& Olshavsky, R. W. (1996). A reexamination of the determinants of consumer satisfaction. J ournal of Marketing, 60, 15-32.

Thibault, J . W., \& Walker, L. (1975). Freedom and reactance New York: Wiley.

Tversky, A. (1977). Features of similarity. Psychological Review, 84, 327- 352.

Tversky, A., \& Gati, I. (1982). similarity, separability, and the triangle inequity. Psychological Review, 89, 123-154.

Tversky, A., \& Shafir, E. (1992). Choice under conflict: The dynamics of deferred decision. Psychol ogical Science, 6, 358-361.

Westbrook, R. A., Newman, J . W., \& Taylor, J . R. (1978). Satisfaction/dissatisfaction in the purchase decision process. J ournal of Marketing, October, 54-60.

Zhang, S., \& Markman, A. (1998). Overcoming early entrant advantage: The role of alignable and nonalignable differences. J ournal of Marketing Research, 35, 413-426.

Zhang, S., \& Sood, S. (1999). Option information as the determinant of choice deferral. Manuscript in preparation.

Received: J une 3, 1998 